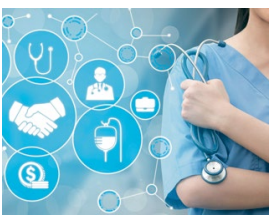

Al's role in COVID-19 epidemiology p1010

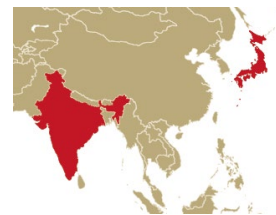

Biotech news from around the world p1013

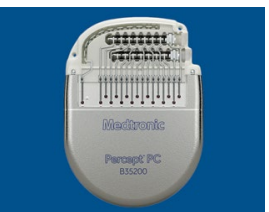

First DBS with neurofeedback approved p1014

\title{
Antibody engineers seek optimal drug targeting TIGIT checkpoint
}

Four antibodies are already in trials to test efficacy against the emerging checkpoint molecule TIGIT, but debate swirls around the importance of Fc binding for drug action.

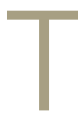

he race to develop a first-in-class checkpoint-blocking drug is heating up, with new clinical data and investor money all buttressing the idea of targeting the co-inhibitory molecule TIGIT (formally known as T cell immunoreceptor with immunoglobulin and ITIM domains) alongside obstruction of the programmed cell death-1 (PD-1) pathway to treat cancer. The combination strategy appears safe and effective against a range of solid tumor types, according to two recent medical meeting reports describing patients treated with Genentech's fully human IgG1 monoclonal antibody $(\mathrm{mAb})$ tiragolumab, the most advanced anti-TIGIT therapy, now in late-stage clinical development.

Others are keeping pace, though, with new funding to test their TIGIT-targeting antibody candidates. In July, for example, iTeos Therapeutics went public, netting \$201 million - on top of $\$ 125$ million in private capital raised earlier in the year - which should allow the company to advance its human anti-TIGIT IgG1 mAb EOS-448 into phase 2 trials. In June, Mereo BioPharma similarly completed a $\$ 70$ million financing round to progress etigilimab, a humanized IgG1 anti-TIGIT $m A b$ previously shown to help stabilize tumor growth and produce a favorable immune profile in patients.

TIGIT is "poised to be the next validated checkpoint," says Dhan Chand, head of drug discovery at Agenus, which itself will soon launch first-in-human trials involving two different anti-TIGIT antibodies, one monoclonal and one bispecific. "If you're going to be in this field - just like with antiPD-1 - you need to have [an anti-TIGIT drug] in your toolbox," adds Terry Rosen, CEO of Arcus Biosciences, which entered into a collaborative development pact with Gilead Sciences in May 2020 centered in large part around TIGIT-directed therapy. Rosen describes TIGIT-directed antibodies

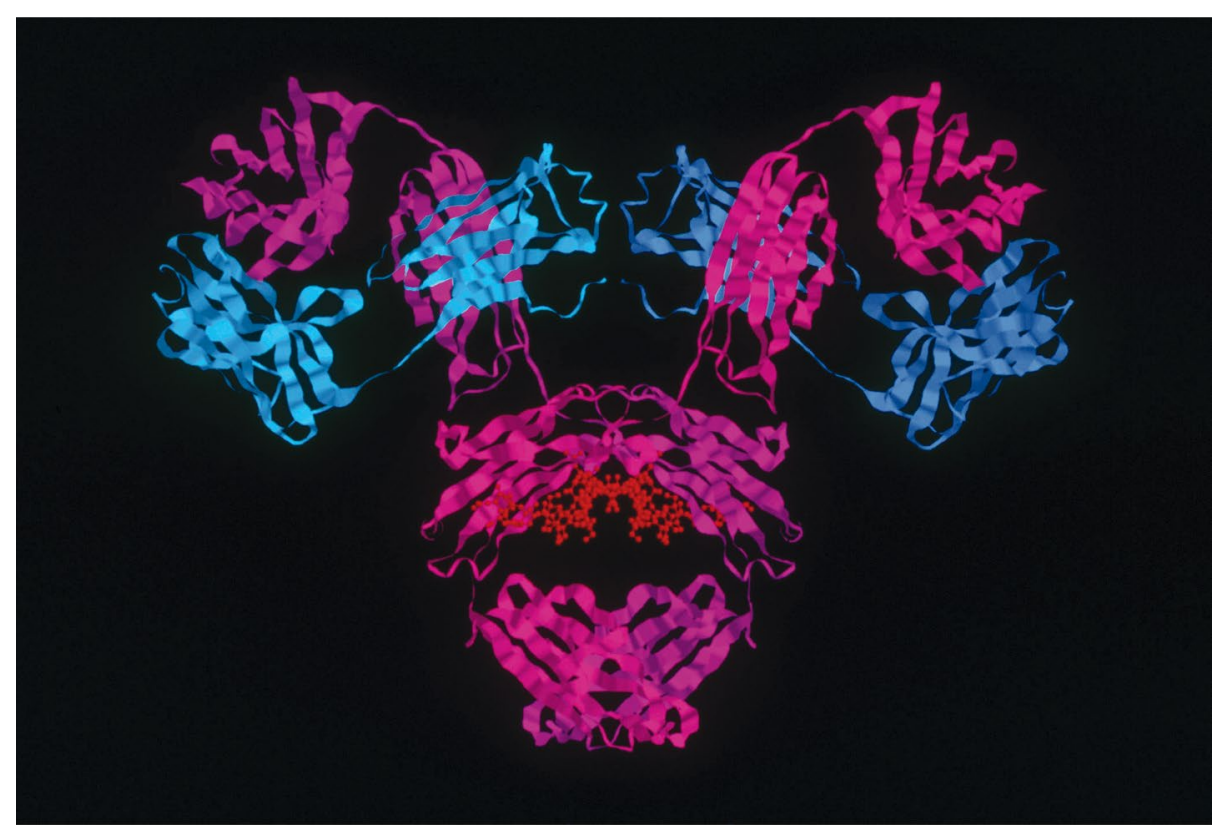

With or without Fc? Drug makers pursuing anti-TIGIT checkpoint inhibitors are undecided whether the Fc portion of an mAb (lower group in picture) is worth keeping intact. Credit: Kenneth Eward / Science Source

as the "potential next backbone therapy" in immuno-oncology.

But even with a dozen candidates now in clinical testing (Table 1), and several others nearing the first-in-human stage, many questions remain about how exactly TIGIT blockade promotes antitumor immunity. Some scientists emphasize the importance of TIGIT signaling in dialing down innate and adaptive immune responses by limiting the effector functions of cytotoxic $\mathrm{T}$ cells and natural killer (NK) cells. Others point to the immunosuppressive action of TIGIT in enhancing the activity of regulatory $\mathrm{T}\left(\mathrm{T}_{\text {reg }}\right)$ cells or in curbing the release of proinflammatory cytokines from dendritic cells.
And whichever mechanism predominates could massively affect the clinical success of the various anti-TIGIT therapies jockeying to reach the marketplace, owing to differences in the $\mathrm{Fc}$ binding capacity of each antibody candidate. "There's so much debate about this $\mathrm{Fc}$ " issue among investors and industry insiders alike, says Daina Graybosch, a research analyst at SVB Leerink who specializes in immuno-oncology drugs. "People keep talking about it."

All anti-TIGIT mAbs in trials today have one key common attribute: they display high levels of target affinity and receptor occupancy. In principle, then, they should each be able to prevent TIGIT from binding 
Table 1 | Anti-TIGIT antibody drugs in clinical development

\begin{tabular}{|c|c|c|c|c|}
\hline Agent & Sponsor & Phase & lgG isotype & Combination immunotherapy \\
\hline Tiragolumab & Roche & 3 & $\lg G 1$ & Anti-PD-L1 (atezolizumab) \\
\hline Vibostolimab & Merck & 2 & $\lg G 1$ & Anti-PD-1 (pembrolizumab) \\
\hline Domvanalimab & Arcus, Gilead & 2 & Mutant IgG1 (Fc receptor disabled) & Anti-PD-1 (zimberelimab); anti-A2aR/A2bR (AB928) \\
\hline BMS-986207 & BMS & $1 / 2$ & Mutant lgG1 (Fc receptor disabled) & Anti-PD-1 (nivolumab) \\
\hline Etigilimab & Mereo & 1 & $\lg G 1$ & Anti-PD-1 (nivolumab) \\
\hline EOS-448 & iTeos & 1 & $\lg G 1$ & Anti-PD-1 ${ }^{a}$ \\
\hline COM902 & Compugen & 1 & $\lg G 4$ & Anti-PVRIG (COM701)a \\
\hline ASP8374 & Astellas & 1 & $\operatorname{lgG} 4$ & Anti-PD-1 (pembrolizumab) \\
\hline SEA-TGT & Seattle Genetics & 1 & IgG1 (Fc enhanced with afucosylation) & Anti-PD-1 (pembrolizumab) \\
\hline BGB-A1217 & BeiGene & 1 & $\lg G 1$ & Anti-PD-1 (tislelizumab) \\
\hline |B|-939 & Innovent & 1 & Not disclosed & Anti-PD-1 (sintilimab) \\
\hline M6223 & EMD Serono & 1 & Not disclosed & TGF- $\beta$ trap/anti-PD-L1 (bintrafusp alfa) \\
\hline
\end{tabular}

${ }^{a}$ Combination immunotherapy planned but not yet in testing

its ligand, CD155, which should help remove the inhibitory signal that impedes $\mathrm{T}$ cells and NK cells from attacking cancers. Plus, because CD155 serves double duty as a ligand for the co-stimulatory molecule CD226 (also known as DNAX accessory molecule-1, or DNAM-1), TIGIT blockade should free up CD155 to engage its alternate receptor, thereby further promoting overall tumor-directed immunity.

Where the mAb candidates noticeably differ is in their Fc regions - and, as Hassane Zarour, a tumor immunologist at the University of Pittsburgh Hillman Cancer Center, points out: "The way you Fc-engineer the antibody is extremely important."

Many, including Genentech's tiragolumab and Merck's humanized mAb vibostolimab, employ a wild-type IgG1 isotype and thus maintain TIGIT-directed antibody-dependent cellular cytotoxicity (ADCC), an immune process that destroys antibody-coated target cells. This could help ensure the therapies do away with pesky $\mathrm{T}_{\text {reg }}$ cells that express high levels of TIGIT and compromise antitumor immunity. But the drugs might also deplete sought-after cytotoxic cells in the process. And not wanting to take that risk, some companies - most notably, Arcus and Bristol-Myers Squibb (BMS) - have opted to use mutated IgG1 tails with inactivated Fc regions. Others, such as Compugen and Astellas Pharma, built their candidates on IgG4 backbones that offer only weak Fc binding.

Meanwhile, antibodies from Seattle Genetics and Agenus were engineered with souped-up Fc activity. The rationale for Fc enhancement - or, at a minimum, maintaining Fc effector function - stems from the fact that, as Chand puts it, “there's more to Fc than just ADCC." In the case of antibodies targeting cytotoxic T-lymphocyte-associated protein 4 (CTLA-4) and TIGIT at least, Chand's team at Agenus showed that intact Fc zones are needed to fuel interactions with dendritic cells, whose $\mathrm{Fc}-\gamma$ receptors (Fc $\gamma$ Rs) latch onto the antibodies and lead to a kind of cellular reprogramming that enhances antigen-specific $\mathrm{T}$ cell responses, independent of any $\mathrm{T}_{\mathrm{reg}}$ cell depletion. "When you promote better FcyR co-engagement, what you end up doing is creating a glue between a $T$ cell and an antigen-presenting cell - and that stabilization of the immune synapse results in much better $\mathrm{T}$ cell activation," explains Chand.

At the 2019 Society for Immunotherapy of Cancer (SITC) annual meeting, a team from Merck Research Laboratories in Boston also reported preclinical data showing that dendritic-cell activation mediated by Fc $\gamma \mathrm{R}$ co-engagement of an antibody to mouse TIGIT induces cytokine and chemokine expression, which in turn promotes immune infiltration into the tumor microenvironment and boosts co-stimulatory immune signaling. Mouse data from Genentech, iTeos and OncoMed Pharmaceuticals (which last year merged with Mereo) all further suggest that Fc binding is integral to the tumor-shrinking activity of anti-TIGIT therapies. "Now, whether [Fc activity] will actually bear out as a clinical differentiator I think remains to be seen," says Cathi Ahearn, vice president of global product strategy for Genentech's oncology business unit.

The only available phase 2 data come from a study of patients with newly diagnosed non-small-cell lung cancer (NSCLC) involving Genentech's tiragolumab combined with the company's Tecentriq (atezolizumab), an Fc-engineered,

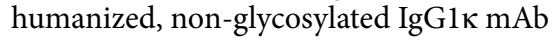
that targets PD-1's binding partner, PD-L1. As reported at the 2020 American Society of Clinical Oncology annual meeting in May, the drug regimen yielded a higher response rate and extended progression-free survival times compared to treatment with atezolizumab alone. Parsing the patients by their tumors' PD-L1 levels, however, showed that the addition of tiragolumab was beneficial only when the immune regulatory molecule was expressed by at least $50 \%$ of cancer cells. In that cohort, tumor shrinkage occurred almost three times as often among patients who received the combination.

To confirm that result, Genentech is now running a registration-enabling trial involving 500 patients with previously untreated NSCLC and PD-L1-high tumors. Another phase 3 trial is evaluating the same combination of anti-TIGIT tiragolumab and anti-PD-L1 Tecentriq, in tandem with induction chemotherapy, in 400 patients with extensive small-cell lung cancer. Smaller trials focused on other tumor types, including cervical cancer and multiple myeloma, are ongoing.

At the same time, Merck is moving ahead with large phase 2 studies of its own anti-TIGIT candidate, vibostolimab, given in combination with other agents, including the PD-1 inhibitor Keytruda (pembrolizumab; a humanized IgG4 $\kappa$ $\mathrm{mAb}$ ) to patients with advanced NSCLC or melanoma. The company has already reported early data from the dose escalation portion of a phase 1 solid tumor study; more results from an expanded cohort of patients with NSCLC are expected in September at the European Society for Medical Oncology virtual congress. 
Taylor Schreiber, CEO of Shattuck Labs, is looking forward to learning those results. If Merck's vibostolimab works about as well as Genentech's tiragolumab, he says, "you can at least make the conclusion that effector competence is not inhibiting the effect of targeting TIGIT - and that is the main thing you'd be worried about." Conversely, if Arcus's humanized IgG1 $\mathrm{mAb}$ domvanalimab, with its Fc-disabled region, shows near-equivalency to the other agents, then it's the TIGIT-blocking function of these antibody therapies that likely matters more than any $\mathrm{Fc}-\mathrm{Fc} \gamma \mathrm{R}$ co-engagement. Initial efficacy data from the domvanalimab trials are anticipated early next year.

For his part, Schreiber is still debating whether to include an Fc-inactive IgG4 linker protein or an afucosylated IgG1 with enhanced Fc activity in Shattuck's TIGIT-directed therapy, a bifunctional fusion protein now in preclinical toxicology testing. He plans to decide after seeing both the Merck and Arcus data. "That will be informative," Schreiber says.

Rosen, the Arcus CEO, isn't too concerned about such comparisons, though. That's because his company is not focused on the same strategy of dual checkpoint inhibition that others are pursuing. On top of TIGIT and PD-1 blockade, Arcus is planning to take a three-pronged attack on tumor immunosuppression with a small molecule drug designed to block signaling of adenosine, an anti-inflammatory metabolite. "That's going to be the place where we bring benefit to patients and provide something that adds to the field," Rosen says. The company is now testing a triplet regimen of its own experimental drugs: the TIGIT inhibitor domvanalimab, the PD-1 inhibitor fully human IgG1 mAb zimberelimab and the small-molecule dual adenosine $\mathrm{A}_{2 \mathrm{~A}} / \mathrm{A}_{2 \mathrm{~B}}$ receptor antagonist etrumadenant in patients with PD-L1-high NSCLC.

In a similar vein, iTeos is planning to evaluate its anti-TIGIT agent, EOS-448, together with its small-molecule $\mathrm{A}_{2 \mathrm{~A}}$ receptor antagonist EOS-850, plus or minus chemotherapy, in the coming year. Likewise, Compugen is positioning its anti-TIGIT therapy, COM902, alongside the company's inhibitor of poliovirus-receptor-related immunoglobulin (PVRIG), a related co-inhibitory receptor that competes with DNAM-1 and TIGIT for binding to the CD112 ligand.

Only by blocking both receptors, PVRIG and TIGIT, can "you actually release the arrest of the system and get sufficient immune stimulation," says Compugen president and CEO Anat Cohen-Dayag. "More than that, there is an intersection between these two pathways and the PD-1 pathway," she adds. "This is a triple pathway story and you need all the relevant pieces in the axis." In phase 1 testing, Compugen's anti-PVRIG drug, COM701, showed early signs of efficacy, both on its own and combined with BMS's PD-1 inhibitor Opdivo (nivolumab). Now, with COM902 still undergoing early clinical testing, Compugen has partnered with BMS to begin evaluating the triple blockade strategy through the combination of COM701, Opdivo and BMS-986207, another anti-TIGIT drug that has already completed the safety portion of a first-in-human study.

Other companies hope to carve out niches in the increasingly crowded anti-TIGIT arena by testing their experimental inhibitors in less common tumor types, or through the use of TIGIT-pathway-specific biomarkers for patient selection. "We're being very careful in exactly how we design these studies," says Mereo CEO Denise Scots-Knight. "We're looking for white space here."

Beyond cancer, some academic groups have also begun to consider situations in which therapeutically augmenting TIGIT activity with an agonistic antibody could be beneficial for patients. Nicole Joller, a T cell immunologist at the University of Zurich in Switzerland, points to the example of severe COVID-19 infections, in which many people experience excessive proinflammatory cytokine storms. "That is a setting where this could be helpful," she says. "You could definitely target TIGIT in anything that's marked by excessive inflammation, be it autoimmune or infection-mediated," Joller notes.

Vijay Kuchroo, from Brigham and Women's Hospital in Boston, says that he and fellow immunologist Dario Vignali, of the University of Pittsburgh School of Medicine, are now shopping around the idea of forming a company focused on advancing checkpoint agonists for treating autoimmunity. Few large drug firms seem to be pursuing this strategy, although Janssen scientists publicly presented in vitro data last year on the immunomodulatory effects of an agonist TIGIT-targeted human IgG1к mAb called TGTB227. According to spokesperson Kellie McLaughlin, the company is "in the process of evaluating the potential of TGTB227 relative to other assets in our portfolio." She declined to comment on future development plans.

\section{Elie Dolgin \\ Somerville, MA, USA}

Published online: 4 September 2020

https://doi.org/10.1038/s41587-020-0666-1

\section{Moderna loses key patent challenge}

A dispute over a key technology used in Moderna's highly anticipated COVID-19 vaccine came to a head in July when Arbutus Biopharma fended off a claim by the vaccine maker. The patent clash over the delivery system used in Moderna's mRNA-1273 vaccine could hamper the biotech's ability to price the vaccine competitively, as well as affect its margins versus those of other companies developing coronavirus vaccines. Moderna previously held a limited sublicense to Arbutus Biopharma's lipid nanoparticle (LNP) formulation, which is used to deliver messenger RNA drugs into cells. But since 2018, Moderna has filed three inter partes reviews (IPRs) with the US Patent and Trademark Office seeking to invalidate Arbutus's LNP patents. The first two IPRs resulted in wins for Moderna - invalidating one of Arbutus's patents in full and partially invalidating another. However, on 23 July the US Patent and Trademark Office's Patent Trial and Appeal Board (PTAB) delivered a decisive win for Arbutus in the third IPR, rejecting Moderna's argument that the Arbutus patent known as the '069 patent should be revoked because it describes obvious concepts. "To the extent it is believed the PTAB erred in their decisions, Moderna may further pursue these matters," the company said in a statement, adding that they were "not aware of any significant intellectual property impediments for any products we intend to commercialize, including mRNA-1273." Though it is still unclear whether Moderna's vaccine development efforts use the LNP technology in question, the PTAB's ruling increases the pressure on Moderna to seek a sublicense on the technology. The '069 patent is licensed to Genevant Sciences, a maker of LNPs for RNA-based therapeutics, and Arbutus would receive $20 \%$ of any revenue received by Genevant for sublicensing the patent. Analysts at investment bank SVB Leerink called the decision a "disappointing turn" for Moderna in a note to investors.

Published online: 4 September 2020 https://doi.org/10.1038/s41587-020-0674-1 